\title{
Lidil
}

Revue de linguistique et de didactique des langues

\section{Le français langue étrangère et seconde. Des paysages didactiques}

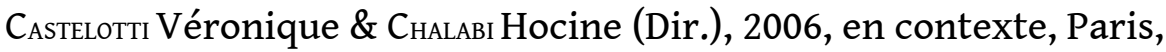
L'Harmattan.

\section{Enrica Piccardo}

\section{(apenEdition}

\section{Journals}

Édition électronique

URL : http://journals.openedition.org/lidil/2303

DOI : 10.4000/lidil.2303

ISSN : 1960-6052

\section{Éditeur}

UGA Éditions/Université Grenoble Alpes

\section{Édition imprimée}

Date de publication : 1 juin 2007

Pagination : 177-182

ISBN : 978-2-84310-100-7

ISSN : $1146-6480$

\section{Référence électronique}

Enrica Piccardo, "Le français langue étrangère et seconde. Des paysages didactiques », Lidil [En ligne], 35 | 2007, mis en ligne le 25 mars 2009, consulté le 24 septembre 2020. URL : http://

journals.openedition.org/lidil/2303; DOI : https://doi.org/10.4000/lidil.2303

Ce document a été généré automatiquement le 24 septembre 2020.

(C) Lidil 


\title{
Le français langue étrangère et seconde. Des paysages didactiques
}

\author{
CAstelotti Véronique \& C
} L'Harmattan.

\section{Enrica Piccardo}

1 Le texte coordonné par Véronique Castellotti et Hocine Chalabi, constitue la suite du colloque qui a eu lieu en 2003 à l'Université François Rabelais de Tours à l'occasion des $90 \ltimes$ ans de vie de l'Institut de Touraine et comprend le texte de toutes les contributions.

2 Le long ouvrage a été organisé autour de cinq axes thématiques, ce qui aide le lecteur à se repérer à travers les vingt-sept articles proposés. Les cinq parties correspondant aux différentes axes sont précédées - en plus de la présentation des deux coordonnateurs par un article de Daniel Coste qui analyse le concept de plurilinguisme et s'interroge sur les enjeux de cette notion et sur ses implications en didactique des langues.

3 Après un aperçu historique visant à la fois à éclaircir la question terminologique liée au plurilinguisme et à réfléchir sur la relation difficile, souvent ambigüe, que la France a entretenue avec son propre paysage plurilingue, l'auteur présente les atouts de la notion de compétence plurilingue, vue non pas comme simple juxtaposition de compétences plus ou moins homogènes dans différentes langues et visant à s'approcher le plus possible de la compétence monolingue d'un parlant natif idéalisé, mais plutôt comme un «aensemble composite, déséquilibré et dynamique de variétés relevant en effet de plusieurs langues, mais géré comme un capital complexe de ressources diversifiées $₫(p . \ltimes 15)$. Dans le scénario varié qu'est la compétence plurilingue, l'inévitable insécurité linguistique finit par constituer un facteur de changement et de progrès. Comment donc - s'interroge D. Coste - transformer cette polyphonie en terrain fertile de construction d'une compétence plurielle qui ne soit pas en dissonance avec la classe de français $\ltimes$ ? Comment dépasser le leurre d'une didactique uniformisante, d'un modèle universel outillé de méthodologies passe-partout ? C'est vers la notion de contexte que l'auteur se tourne pour développer sa réflexion. Et il part de la polysémie acquise par ce terme en didactique des langues pour questionner le rôle de cette dimension en tant que levier de renouvellement méthodologique. Sa 
conclusion, qui est en même temps ouverture à la thématique du colloque, plaide pour une utilisation de toute diversité comme occasion de prise en compte des contextes, non pas pour une soumission ou un relativisme didactique dépourvu de toute règle, mais plutôt pour un recadrage et une réflexion transversale forte capables de faire évoluer les contextes mêmes où on est appelé à intervenir.

Le texte se poursuit avec la première partie qui regroupe des interventions concernant la problématique de la relation offre-demande et les politiques linguistiques - et options éducatives conséquentes - qui sont liées à cette problématique.

Les contributions relatent des spécificités de la situation des Pays-Bas, de la Chine, de l'Algérie, de l'Espagne, des îles Fidji et du Ghana ; en plus de cela, il y a une contribution portant sur la situation particulière qui est celle des sections bilingues où on réfléchit sur le statut du français en tant que langue d'apprentissage.

6 Les articles font un état des lieux de la place du français dans les différents pays en montrant la grande variété des facteurs qui entrent en jeu dans la dynamique du choix de cette langue. Les articles qui s'articulent autour d'une perspective de type descriptif et/ou historique visant à expliquer les spécificités de chaque pays, s'interrogent sur quelques aspects d'ordre sociologique liés à la perception du français, de sa place et de son rôle. Des problèmes tels que la non considération des réalités locales émergent clairement. Le plurilinguisme des Iles Fiji n'est pas pris en compte, alors qu'il pourrait constituer une vraie richesse à valoriser et à l'intérieur de laquelle l'étude du français pourrait trouver sa place. Il en va de même du positionnement ambivalent de la population ghanéenne envers cette langue, objet à la fois désiré pour le capital symbolique que son usage procure et rejeté car son appropriation s'apparente à un double reniement de l'identité africaine.

7 Si une partie des contributions se limite à présenter la réalité de l'enseignement du FLE dans les différents pays ou la spécificité du français dans les classes bilingues en Europe, en dénonçant au plus les problèmes ou les manques de cohérence, d'autres cherchent à se poser des questions visant à faire évoluer la situation, notamment en matière de politique d'enseignement et de formation des enseignants. C'est le cas de l'article de Carmen Guillén Diaz concernant le FLE dans le système éducatif espagnol, qui propose un éventail de pistes vraiment ouvertes à l'interdisciplinarité et au plurilinguisme.

8 La deuxième partie s'interroge sur le rôle du français en tant que langue d'insertion aussi bien en contexte scolaire que hors de la classe.

9 La thématique complexe est introduite par une contribution de Jean-Louis Chiss et suivie par deux contributions qui relatent respectivement des pratiques en contexte français hexagonal et en Belgique francophone.

10 L'article de Chiss se concentre sur la notion de français langue seconde en France et mentionne les dispositifs institutionnels qui ont permis des avancées en termes de reconnaissance statutaire de son enseignement et de réflexion dans la dimension didactique de cette langue et de toute langue en général. En partant de ces considérations sur le scénario actuel du FLS, il s'interroge sur le rôle potentiel du FLS vis-à-vis du dépassement de la tradition monolingue française. Il introduit des notions de type transversal telles que celle de «xliteracy» (littératie), concernant le rôle de structuration de la pensée et de codification des savoirs joué par l'écriture et souligne l'importance qu'un travail explicite sur cette notion aurait sur un milieu multilingue tel 
qu'une classe constituée, entre autre, d'enfants issus de l'immigration. Ce travail métalinguistique est aussi important par rapport à l'oral car une attention particulière à la manière dont les échanges linguistiques structurent les apprentissages s'avère nécessaire. Il faut donc envisager une étude du profil de l'élève nouvellement arrivé en France et les langues ont un rôle clé à jouer par rapport à cela aussi bien du point de vue socioculturel que du point de vue psycholinguistique. Le FLS serait à la fois un banc d'essai pour la faisabilité des démarches inter- ou pluriculturelles et un observatoire privilégié des stratégies des apprenants.

11 Deux exemples suivent qui montrent à la fois la richesse d'une démarche qui prend en compte la multiplicité des cultures et des langues de la classe et qui les intègre dans le processus d'enseignement/apprentissage en la valorisant et de l'autre les difficultés dues au manque d'une coordination et d'une ingénierie éducative ciblées, tout comme les carences de formation adaptée des enseignants appelés à intervenir dans des nouveaux dispositifs.

12 La troisième partie relate différentes adaptations. Il est question de voir le contexte en termes de dynamique entre cultures d'apprenants et répertoires d'enseignants. Pour ce qui est du premier point, des contributions nous montrent des aspects révélateurs de la relation que des apprenants japonais ont avec l'apprentissage du FLE, quels clivages existent entre motivation des apprenants et enseignement offert, quels facteurs contextuels scolaires et extrascolaires interviennent, comment les représentations se construisent et se modifient.

13 La spécificité de la situation thaïlandaise fait aussi l'objet d'une contribution. On revient ensuite au contexte hexagonal, et on s'interroge sur les modalités d'enseignement de la littérature et sur le fait que cet enseignement n'est pas universel, mais plutôt très fortement connoté du point de vue culturel.

On passe ensuite à la dimension enseignante, avec quatre contributions qui s'interrogent sur des notions telles que la posture réflexive en situation d'analyse des pratiques, la notion de rythme et de " naturel didactique ", la formation à l'autoobservation participante, la nécessité d'un travail profond d'activation des représentations des acteurs impliqués dans le processus d'enseignement/apprentissage à travers un travail ciblé sur les métadiscours de la classe.

15 Les orientations méthodologiques font l'objet de la quatrième partie de l'ouvrage. Les quatre contributions qui composent cette partie abordent des aspects particuliers liés à la didactique du FLE. Il est question de s'interroger sur le rôle de la grammaire, en particulier de la "grammaire instructionnelle » dans un contexte multiple tel que celui des classes actuelles. L'exceptionnalité de classes linguistiquement homogènes, au lieu d'être perçue comme un obstacle, pourrait constituer un point de départ pour que l'enseignement du français devienne d'autant plus efficace que réfléchi. La notion de genre discursif en FLE est ensuite interrogée, non pas en tant que phénomène de mode, mais pour son apport au niveau didactique. La contribution reprend historiquement la question des genres de discours à partir du Niveau-Seuil jusqu'au CECR pour montrer ses potentialités en tant que notion passerelle entre enseignement communicatif et enseignement centré sur les tâches. Les deux autres contributions interrogent certaines implications de la notion de contextes à partir de manuels, respectivement une méthode de FLE en Jordanie et un manuel de français tunisien pour la classe terminale, en montrant tous les manques par rapport à la prise en compte de la dimension socio-culturelle des pays où ces manuels sont employés. 
L'ouvrage se termine avec une cinquième partie portant sur les problématiques liées à l'enseignement $d u$ français sur objectifs spécifiques. Dans ce domaine la notion d'adaptabilité aux contextes s'avère capitale comme le montrent les quatre contributions. La prise en compte de la dimension culturelle apparait essentielle si on veut éviter un enseignement neutre et dépourvu de toute considération pour le facteur humain. Qu'il s'agisse de centres universitaires de FLE, d'enseignement dyadique finalisé à la mobilité personnelle dans les entreprises, d'enseignement pour des masters très spécialisés ou, plus généralement, de la conception d'un programme de formation au FOS et du manuel spécifique, les exigences d'un enseignement sur objectifs spécifiques soulignent certaines notions qui ont traversé tout le colloque et sur lesquelles les différentes contributions ont cherché à apporter un regard clarificateur. La prise en compte des représentations, des styles d'enseignement et des styles d'apprentissage, de la variation culturelle et de la dimension culturellement connotée de la communication, la notion surtout d'adaptation qui requiert des comportements qui relèvent presque de l'ethnométhodologie ne font que souligner une fois de plus le besoin de formation à la complexité, à la diversité, aux savoir-faire culturels et in fine à la valorisation de cette profonde richesse.

\section{AUTEUR}

\section{ENRICA PICCARDO}

Université Stendhal-Grenoble 3, Lidilem. 